\title{
Increased TRPC3 expression in vascular endothelium of patients with malignant hypertension
}

\author{
Florian Thilo ${ }^{1,3}$, Christoph Loddenkemper ${ }^{2,3}$, Erika Berg ${ }^{2}$, Walter Zidek ${ }^{1}$ and Martin Tepel ${ }^{1}$ \\ ${ }^{1}$ Medizinische Klinik, Nephrologie, Charité Campus Benjamin Franklin, Berlin, Germany and ${ }^{2}$ Institut für \\ Pathologie, Charité Campus Benjamin Franklin, Berlin, Germany
}

\begin{abstract}
An increased expression of transient receptor potential canonical type 3 (TRPC3) cation channels has been proposed as one of the factors contributing to the pathogenesis of hypertension. To test that hypothesis we compared the expression of TRPC3 and TRPC6 as an endogenous control in human vascular endothelium of preglomerular arterioles in kidney biopsies from six patients with malignant hypertension and from four patients with diarrhea-associated hemolytic-uremic syndrome. Patients with malignant hypertension showed significantly higher systolic blood pressure and more prominent expression of TRPC3 in vascular endothelium of preglomerular arterioles compared to patients with hemolytic-uremic syndrome. The expression of TRPC6 was not different between the two groups. The study supports the hypothesis that the increased expression of TRPC3 is associated with malignant hypertension in humans.
\end{abstract}

Modern Pathology (2009) 22, 426-430; doi:10.1038/modpathol.2008.200; published online 9 January 2009

Keywords: transient receptor potential channel; malignant hypertension; vascular endothelium

Malignant hypertension is a hypertensive emergency with severe elevation of blood pressure, a Keith-Wagener-Barker grade III or IV hypertensive retinopathy, and acute impairment of renal function in most cases. In white subjects, essential hypertension accounts for approximately $30 \%$ of malignant hypertension, whereas in blacks essential hypertension is the predominant cause, accounting for approximately $82 \%$ of all cases. The characteristic pathological change of malignant hypertension represents endothelial injury. Its pathophysiology involves mechanical stress on the vessel wall, a proliferative endarteriitis in small arteries and arterioles, and fibrinoid necrosis with fine subendothelial droplets and hyalin thrombi formation. ${ }^{1,2}$

Recently, evidence had been obtained that an increased expression of nonselective transient receptor potential canonical type 3 (TRPC3) cation channels contributes to the pathogenesis of hypertension. Liu et $a l^{3}$ reported increased TRPC3 protein expression in spontaneously hypertensive rats

Correspondence: Dr M Tepel, Medizinische Klinik, Nephrologie, Charité Campus Benjamin Franklin, Hindenburgdamm 30, Berlin 12200, Germany.

E-mail: Martin.Tepel@charite.de

${ }^{3}$ These authors contributed equally to this work.

Received 26 August 2008; accepted 7 November 2008; published online 9 January 2009 compared to normotensive Wistar-Kyoto rats. Dietrich et $a l^{4}$ reported that TRPC6-deficient mice with consecutively increased TRPC3 channel expression developed higher blood pressure. Liu et $a l^{5,6}$ and Thilo et $a l^{7}$ reported increased TRPC3 protein expression and increased TRPC3 transcripts in peripheral blood monocytes from patients with essential hypertension compared to normotensive control subjects. The expression of TRPC3 in human vascular endothelium has not been associated with malignant hypertension yet. We designed this study to test the hypothesis that the expression of TRPC3 in human vascular endothelium of preglomerular arterioles is associated with malignant hypertension. To this end, we compared the expression of TRPC3 in human vascular endothelium of preglomerular arterioles in kidney biopsies from patients with malignant hypertension and from patients with diarrhea-associated hemolytic-uremic syndrome.

\section{Methods}

Kidney biopsies from patients who had acute renal insufficiency and who met the inclusion criteria were used in this study. Kidney biopsies were obtained from January 1998 to December 2007 and analyzed at the Institut für Pathologie, Charite Campus Benjamin Franklin, Berlin, Germany. The 
Table 1 Characteristics of the patients

\begin{tabular}{|c|c|c|c|c|c|c|c|c|c|}
\hline $\begin{array}{l}\text { Variable } \\
\text { number }\end{array}$ & $\begin{array}{c}\text { Age } \\
\text { (years) }\end{array}$ & Sex & $\begin{array}{l}\text { Prodromal } \\
\text { diarrhea }\end{array}$ & $\begin{array}{c}\text { Body mass } \\
\text { index } \\
\left(\mathrm{kg} / \mathrm{m}^{2}\right)\end{array}$ & $\begin{array}{l}\text { Systolic } \\
\text { blood } \\
\text { pressure } \\
\text { (mm Hg) }\end{array}$ & $\begin{array}{l}\text { Diastolic } \\
\text { blood } \\
\text { pressure } \\
\text { (mm Hg) }\end{array}$ & $\begin{array}{l}\text { Heart rate } \\
\text { (per min) }\end{array}$ & $\begin{array}{c}\text { GFR }(\mathrm{ml} / \mathrm{min} \\
\left.\text { per } 1.72 \mathrm{~m}^{2}\right)\end{array}$ & $\begin{array}{c}\text { Platelet } \\
\text { count } \\
\text { (per nl) }\end{array}$ \\
\hline \multicolumn{10}{|c|}{ Patients with malignant hypertension } \\
\hline 1 & 40 & $\mathrm{M}$ & No & 27.0 & 200 & 100 & 72 & 4 & 104 \\
\hline 2 & 45 & $\mathrm{M}$ & No & 25.3 & 240 & 120 & 66 & 5 & 294 \\
\hline 3 & 42 & $\mathrm{M}$ & No & 30.4 & 166 & 90 & 82 & 42 & 106 \\
\hline 4 & 35 & $\mathrm{M}$ & No & 26.2 & 240 & 160 & 115 & 4 & 218 \\
\hline 5 & 36 & $\mathrm{~F}$ & No & 22.3 & 200 & 130 & 84 & 14 & 155 \\
\hline 6 & 40 & $\mathrm{M}$ & No & 22.1 & 240 & 140 & 61 & 37 & 229 \\
\hline Median & 40 & - & - & 25.8 & $220^{*}$ & 125 & 77 & 9 & 186 \\
\hline $\begin{array}{l}25 \text { th-75th } \\
\text { percentiles }\end{array}$ & $36-43$ & - & - & $22.3-27.9$ & $191-240$ & 98-145 & $65-92$ & $4-38$ & $105-245$ \\
\hline \multicolumn{10}{|c|}{ Patients with hemolytic-uremic syndrome } \\
\hline 1 & 58 & $\mathrm{M}$ & Yes & 21.5 & 180 & 122 & 140 & 11 & 41 \\
\hline 2 & 24 & $\mathrm{~F}$ & Yes & 26.0 & 160 & 85 & 125 & 5 & 40 \\
\hline 3 & 5 & $\mathrm{~F}$ & Yes & 20.8 & 184 & 122 & 100 & 16 & 202 \\
\hline 4 & 42 & $\mathrm{M}$ & Yes & 26.0 & 130 & 70 & 88 & 17 & 76 \\
\hline Median & 33 & - & - & 23.7 & 170 & 104 & 112 & 13 & 59 \\
\hline $\begin{array}{l}25 \text { th-75th } \\
\text { percentiles }\end{array}$ & $10-45$ & - & - & $21.0-26.0$ & $138-184$ & $74-125$ & $91-136$ & $7-17$ & $40-171$ \\
\hline
\end{tabular}

GFR denotes estimated glomerular filtration rate according to the modification of diet in renal disease formula.

${ }^{*} P<0.05$ for the comparison with the patients with hemolytic-uremic syndrome.

indication to perform a kidney biopsy was determined by each patient's physician for evaluation of the diagnosis of acute renal insufficiency. Informed consent for kidney biopsies was obtained from each patient.

Inclusion criteria comprised malignant hypertension or diarrhea-associated hemolytic-uremic syndrome. The diagnosis malignant hypertension was verified according to the World Health Organization criteria of 1978: high blood pressure together with bilateral linear or flame-shaped hemorrhages or 'cotton-wool' exudates with or without papilledema during funduscopic examination (grade III or IV hypertensive retinopathy, respectively, according to the Keith-Wagener-Barker classification). ${ }^{2}$ The diarrhea-associated hemolytic-uremic syndrome was defined by the presence of the following. ${ }^{8,9}$ Prodromal diarrhea, microangiopathic hemolytic anemia, ie a hemoglobin concentration below $10 \mathrm{~g}$ per $100 \mathrm{ml}$ and nonimmune hemolysis with fragmented red cells or schistocytes on a peripheral blood smear, increased serum lactate dehydrogenase, reduced serum haptoglobin, thrombocytopenia (a platelet count of less than 150 per nl), and acute renal insufficiency (a reduced glomerular filtration rate less than $30 \mathrm{ml} / \mathrm{min}$ per $1.72 \mathrm{~m}^{2}$ ) without prior history of chronic renal insufficiency. The period of risk for the development of the hemolytic-uremic syndrome was considered to be 14 days after the onset of diarrhea according to data from the literature. ${ }^{9}$

Exclusion criteria were pregnant women, patients with papilledema and an intracranial hemorrhage, patients already on dialysis before admission, or evidence of renal parenchymal disease including chronic glomerulonephritis, chronic pyelonephritis, diabetic nephropathy, or tubulointerstitial disease on histologic examination of kidney biopsies. Patients with significant renal artery stenosis as defined if narrowing of the renal artery lumen exceeded $50 \%$ or patients with the diagnosis of pheochromcytoma were excluded. Patients with a diagnosis of thrombotic thrombocytopenic purpura based on clinical evaluation and laboratory findings including deficiency of the metalloprotease a disintegrin and metalloprotease domain with thrombospondin type1 motif13 (ADAMTS13) or inhibitory anti-ADAMTS13 antibodies were excluded.

\section{Immunohistochemistry}

The expression of TRPC3 or TRPC6 for control in human vascular endothelium of preglomerular arterioles in kidney biopsies was analyzed by immunohistochemistry using formalin-fixed paraffin-embedded tissue. Sections ( $4 \mu \mathrm{m}$ thick) were cut, deparaffinized, and subjected to heatinduced epitope retrieval step before incubation with antibodies. For this purpose, sections were immersed in sodium citrate buffer at $\mathrm{pH} 6.0$ and heated in a high-pressure cooker. After cooking, the slides were rinsed in running water, washed with Tris-buffered saline, pH 7.4 and incubated with the primary rabbit anti-TRPC3, or rabbit anti-TRPC6 antibodies (dilution 1:1000; Alomone Labs, Jerusalem, Israel) diluted in 1\% bovine serum albumin in phosphate-buffered saline. Previously published immunoblottings from our group already confirmed that these antibodies can be used to identify TRPC3 

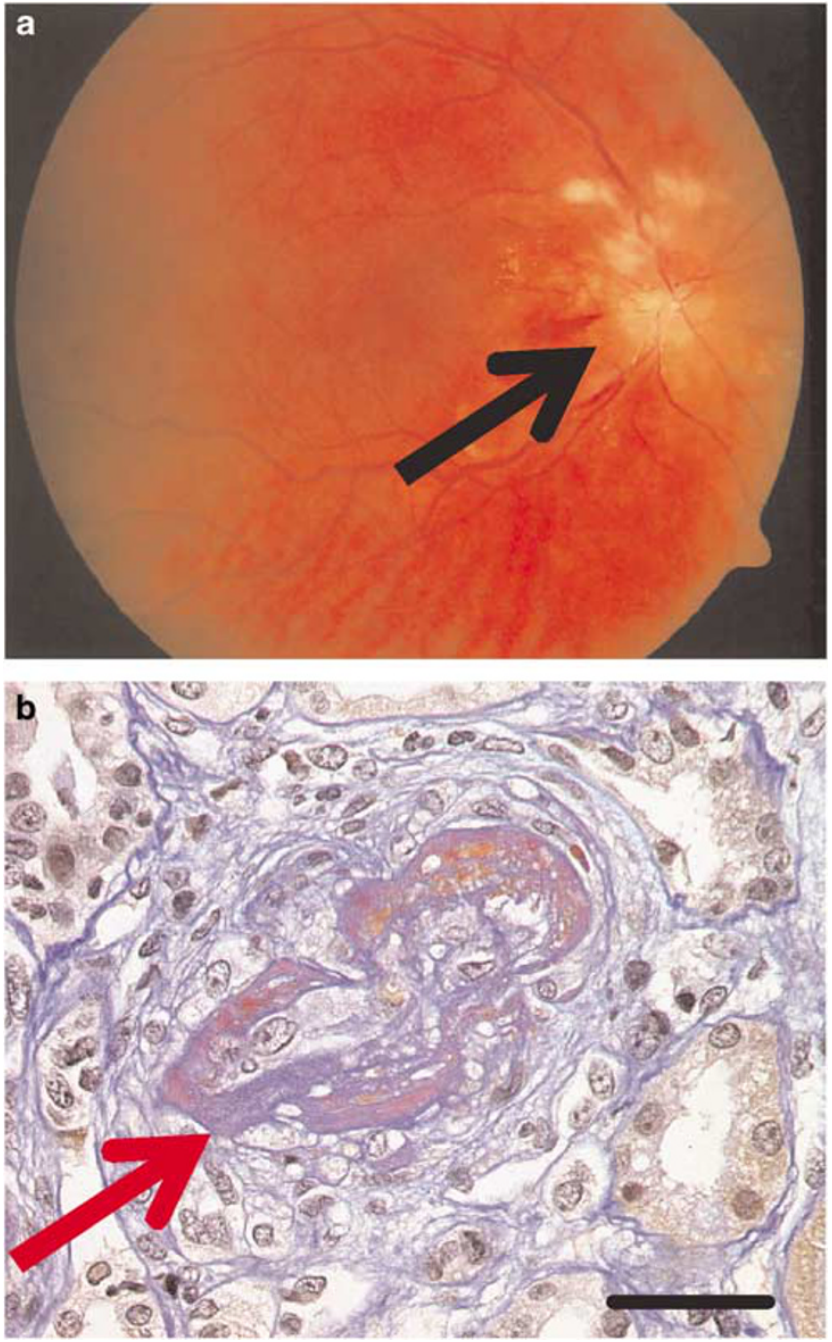

Figure 1 (a) Representative funduscopic examination of one patient with malignant hypertension showing flame-shaped hemorrhages or 'cotton-wool' exudates with papilledema (grade IV hypertensive retinopathy according to the Keith-WagenerBarker classification; arrow). (b) Representative light micrograph showing fibrinoid necrosis (arrow) in a preglomerular arteriole in one patient with malignant hypertension. Fibrin staining. Bar denotes $10 \mu \mathrm{m}$.

or TRPC6 channels in human tissue. ${ }^{5,6}$ The streptavidin AP kit (K5005; Dako, Glostrup, Denmark) was used for detection and alkaline phosphatase was developed using Fast Red as the chromogen. After counterstaining with hematoxylin, the slides were dehydrated and mounted. Negative controls were performed by omitting the primary antibodies. Intensity of TRPC3 or TRPC6 expression levels in the human vascular endothelium of preglomerular arterioles was scored as negative $(-)$, weak $(+)$, moderate $(++)$, or strong $(+++)$. Immunohistochemistry was independently evaluated by two investigators that were unaware of the patients' disease status and in case of discrepancy slides were discussed over a multihead microscope until consensus was reached.

\section{Statistics}

All data were expressed as medians and interquartile ranges. The Mann-Whitney test was used to compare data from the two groups. The null hypothesis was rejected at $P<0.05$. All tests were two sided. Analysis was performed using GraphPad Prism version 5.00 for Windows, GraphPad Software, San Diego, CA, USA.

\section{Results}

The clinical and biochemical characteristics of six patients with malignant hypertension and four patients with diarrhea-associated hemolytic-uremic syndrome are depicted in Table 1. Systolic blood pressure and platelet count were significantly higher in patients with malignant hypertension than in patients with hemolytic-uremic syndrome $(P<0.05)$, whereas age, body mass index, diastolic blood pressure, heart rate, and glomerular filtration rate were not significantly different between the two groups.

Figure 1a depicts the typical funduscopic examination of one patient with malignant hypertension showing flame-shaped hemorrhages or 'cotton-wool' exudates with papilledema (grade IV hypertensive retinopathy according to the Keith-Wagener-Barker classification). In patients with malignant hypertension kidney biopsy revealed acute hypertensive injury. A representative light micrograph showing characteristic obliterative vasculopathy due to endothelial damage and fibrinoid necrosis in a preglomerular arteriole in one patient with malignant hypertension is depicted in Figure 1b. In patients with malignant hypertension histologic examination of kidney biopsies did not give evidence of renal parenchymal disease including chronic glomerulonephritis, chronic pyelonephritis, diabetic nephropathy, or tubulointerstitial disease.

Now we evaluated whether elevated blood pressure was associated with increased expression of TRPC3 in human vascular endothelium of preglomerular arterioles using immunohistochemistry. As indicated in Figure 2 patients with malignant hypertension showed more prominent expression of TRPC3 in preglomerular arterioles compared to patients with hemolytic-uremic syndrome. We observed a grade +++ expression of TRPC3 in five out of six patients with malignant hypertension, and one patient with malignant hypertension showed a grade ++ expression of TRPC3, whereas only two out of four patients with diarrheaassociated hemolytic-uremic syndrome showed a grade + expression and two patients showed a grade - expression (Table 2). The expression of TRPC6 was not different between the two groups.

\section{Discussion}

Our findings, obtained in a consecutive series of patients who had acute renal insufficiency due to 


\section{PAS}
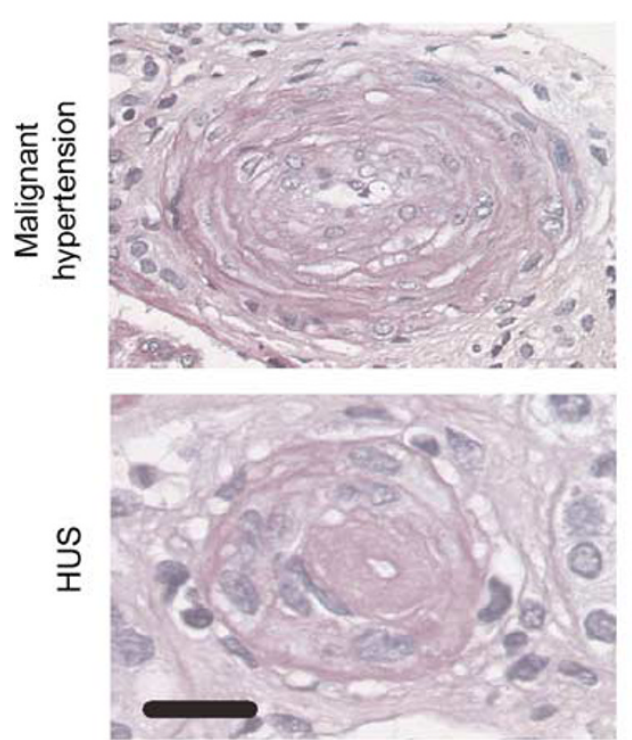

TRPC3
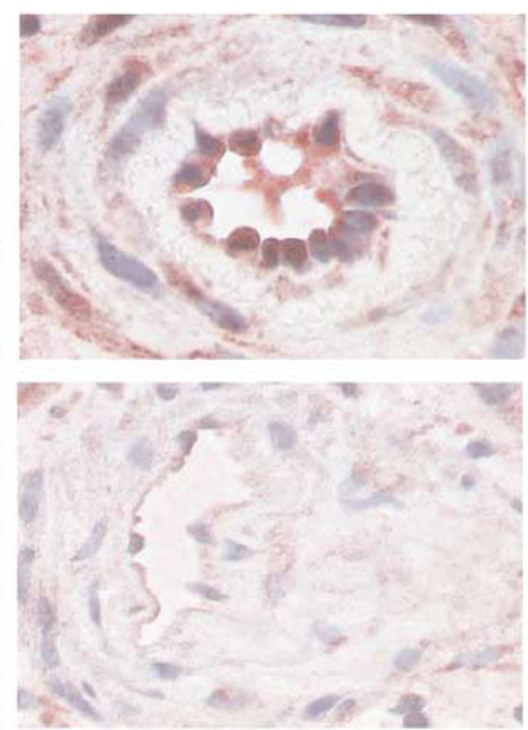

TRPC6
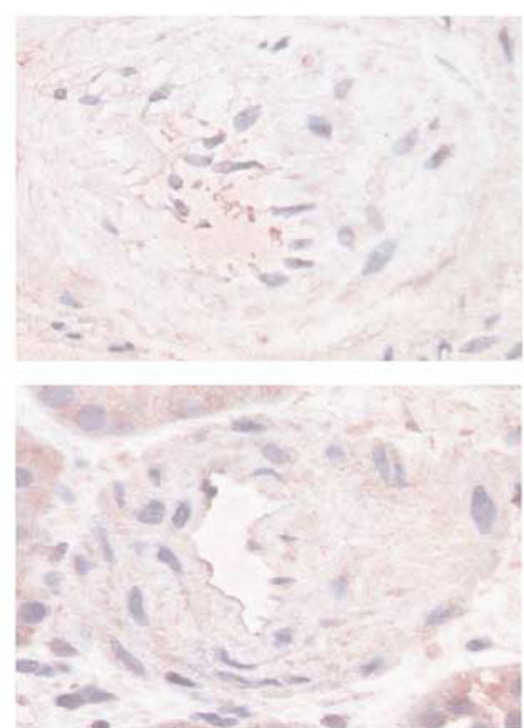

Figure 2 Representative light micrographs and immunohistochemistry of human vascular endothelium of preglomerular arterioles in a patient with malignant hypertension showing onion -skin changes and thickening of the intima (upper panel) and a patient with diarrhea-associated hemolytic-uremic syndrome (lower panel). PAS, periodic acid schiff staining; TRPC3, transient receptor potential canonical type 3; TRPC6, transient receptor potential canonical type 6 . Prominent expression of TRPC3 (grade +++ ) in human vascular endothelium from the patient with malignant hypertension can be observed. Bar denotes $10 \mu \mathrm{m}$.

Table 2 Immunohistochemistry

\begin{tabular}{crc}
\hline Variable number & TRPC3 expression & TRPC6 expression \\
\hline Patients with malignant hypertension & \\
1 & +++ & - \\
2 & +++ & - \\
3 & +++ & + \\
4 & ++ & - \\
5 & +++ & + \\
6 & +++ & \\
& & + \\
Patients with hemolytic-uremic syndrome & - \\
1 & + & - \\
2 & - & - \\
3 & - & \\
4 & + & \\
\end{tabular}

Scores can range from - to +++; higher scores indicate more severe expression.

malignant hypertension, supports the hypothesis that the increased expression of TRPC3 channels in human vascular endothelium of preglomerular arterioles is associated with hypertension in humans. In patients with malignant hypertension funduscopic examination showed characteristic grade IV hypertensive retinopathy and kidney biopsies showed characteristic obliterative vasculopathy due to endothelial damage. ${ }^{1}$ The choice of appropriate controls is important for investigations of malignant hypertension. We compared patients with malignant hypertension and patients with diarrhea-associated hemolytic-uremic syndrome. On the one hand both diseases are associated with acute renal insufficiency and endothelial injury in renal arterioles. On the other hand the underlying pathogenesis is different for both diseases. In malignant hypertension severely increased blood pressure is the main contributing factor, whereas in diarrhea-associated hemolytic uremic syndrome mainly bacterial endotoxins trigger vascular injury. $^{10,11}$ As expected this study confirmed that systolic blood pressure and platelet counts were significantly higher in patients with malignant hypertension than in patients with hemolyticuremic syndrome. This result is in accordance with findings in the literature suggesting that severely elevated systolic blood pressure (ie more than $200 \mathrm{~mm} \mathrm{Hg}$ ) and higher platelet counts can be observed in patients with malignant hypertension. ${ }^{1,11}$ Severely elevated systolic blood pressure and higher platelet counts have therefore been proposed to distinguish malignant hypertension from diarrhea-associated hemolytic-uremic syndrome. ${ }^{11}$ The present finding of increased TRPC3 expression in human vascular endothelium in patients with malignant hypertension may also be a helpful diagnostic tool to distinguish both diseases. No data are available yet that linked the expression of TRPC3 in human vascular endothelium of preglomerular arterioles with malignant hypertension. We compared kidney biopsies from patients who had acute renal insufficiency due to malignant hypertension or diarrhea-associated hemolytic-uremic syndrome and hence we had the extraordinary opportunity to connect TRPC3 expression in human vascular endothelium with hypertension. Malignant hypertension is a rare disease and its pathogenesis remains elusive. However, essential 
hypertension appears to be an important underlying factor. Therefore, the observation that TRPC3 expression is increased in vascular endothelium from patients with malignant hypertension may also indicate that an increased expression of TRPC3 channels may be important in the pathogenesis of hypertension. Previous studies from several groups already gave evidence for the association of TRPC3 and essential hypertension. These studies using animal models of primary hypertension or peripheral blood cells from humans reported an increased expression of TRPC3 channel transcripts, increased TRPC3 channel proteins, and increased TRPC3 activation in hypertension. ${ }^{3-7}$ TRPC3 channels are nonselective cation channels mediating calcium influx. The acute calcium influx through TRPC3 channels is a key signaling mechanism that stimulates the calcium-dependent release of several endothelium-derived vasoconstrictive agents including endothelin, urotensin, or epoxyeicosatrienoic acids. ${ }^{12-14}$

In conclusion for the first time we observed direct evidence that TRPC3 expression in human vascular endothelium is associated with malignant hypertension.

\section{References}

1 Kitiyakara C, Guzman NJ. Malignant hypertension and hypertensive emergencies. J Am Soc Nephrol 1998;9:133-142.

2 van den Born BJ, Honnebier UP, Koopmans RP, et al. Microangiopathic hemolysis and renal failure in malignant hypertension. Hypertension 2005;45:246-251.

3 Liu D, Scholze A, Zhu Z, et al. Increased transient receptor potential channel TRPC3 expression in spontaneously hypertensive rats. Am J Hypertens 2005;18:1503-1507.
4 Dietrich A, Mederos Y, Schnitzler M, et al. Increased vascular smooth muscle contractility in TRPC6-/mice. Mol Cell Biol 2005;25:6980-6989.

5 Liu D, Scholze A, Zhu Z, et al. Transient receptor potential channels in essential hypertension. J Hypertens 2006;24:1115-1124.

6 Liu DY, Thilo F, Scholze A, et al. Increased storeoperated and 1-oleoyl-2-acetyl-sn-glycerol-induced calcium influx in monocytes is mediated by transient receptor potential canonical channels in human essential hypertension. J Hypertens 2007;25: 799-808.

7 Thilo F, Scholze A, Liu DY, et al. Association of transient receptor potential canonical type 3 (TRPC3) channel transcripts with proinflammatory cytokines. Arch Biochem Biophys 2008;471:57-62.

8 Chandler WL, Jelacic S, Boster DR, et al. Prothrombotic coagulation abnormalities preceding the hemolyticuremic syndrome. N Engl J Med 2002;346:23-32.

9 Tarr PI, Neill MA, Clausen CR, et al. Escherichia coli O157:H7 and the hemolytic uremic syndrome: importance of early cultures in establishing the etiology. J Infect Dis 1990;162:553-556.

10 Ruggenenti P, Noris M, Remuzzi G. Thrombotic microangiopathy, hemolytic uremic syndrome, and thrombotic thrombocytopenic purpura. Kidney Int 2001;60:831-846.

11 Shibagaki Y, Fujita T. Thrombotic microangiopathy in malignant hypertension and hemolytic uremic syndrome (HUS)/thrombotic thrombocytopenic purpura (TTP): can we differentiate one from the other? Hypertens Res 2005;28:89-95.

12 Fleming I, Busse R. Endothelium-derived epoxyeicosatrienoic acids and vascular function. Hypertension 2006;47:629-633.

13 Watanabe T, Kanome T, Miyazaki A, et al. Human urotensin II as a link between hypertension and coronary artery disease. Hypertens Res 2006;29:375-387.

14 Thijssen DH, Rongen GA, Smits P, et al. Physical (in)activity and endothelium-derived constricting factors: overlooked adaptations. J Physiol 2008;586:319-324. 